\title{
Selection of Wavelength Calibration Features for Automatic Format Recovery in Astronomical Spectrographs
}

\author{
Paul Bristow and Florian Kerber \\ ESO - ESO, Karl-Schwarzschild-Str. 2, 85748 Garching, Germany;
}

\begin{abstract}
Automated wavelength calibration requires line lists that are free of blended or unstable features at the resolution of the spectrograph in order to avoid false matches. We present a procedure that, given a high quality calibration source spectrum as input, will identify isolated features suitable for use as wavelength standards. The procedure takes into account the expected signal to noise level and the degree of uncertainty in the spectral format that defines how isolated the spectral features must be. Our goal is to produce a customised list of features that can be unambiguously matched to observed features.
\end{abstract}

Keywords: Spectroscopy, Wavelength Calibration, Instrument Modelling

\section{INTRODUCTION}

Crucial to the accurate wavelength calibration of spectrographs are the reference wavelengths used. Naturally one must first choose an appropriate source as discussed in Kerber et al, ${ }^{1}$ but here we assume that a good source is available and consider the selection of calibration features from that source. Not only is it important to have an accurate and comprehensive catalogue for the calibration source used, but the subset of calibration features chosen for use with a given instrument is also critical. Features need to be chosen so that:

- They will not be blended at the resolution of the instrument in question.

- They are bright enough to be seen in realistic calibration exposures.

- They provide adequate coverage (baseline and density) across the wavelength range, detector co-ordinates and spectral orders.

This represents a customisation of the reference catalogue to suit a given instrument and operating strategy.

It is easy to think of simple ways to "clean" the catalogue such as selecting only features brighter than some threshold, or only those that have no neighbours within a certain threshold distance. However there are obvious drawbacks too: brighter features just above a certain threshold may have near neighbours that are only just below it; the deeper and more comprehensive the input catalogue, the more crowded faint features in close proximity to bright features. Clearly what is needed is a well defined method for deciding which lines will provide accurately measurable centroids when used with a given instrument.

Fitting a wavelength solution is usually an iterative, bootstrapped process, ideally the line list should be customised to reflect the stages of this process. For example the first stage may involve recovering the approximate wavelength solution and spectral format of the instrument after some physical change in the instrument (eg. maintenance or upgrade interventions or earthquake damage). In this situation the locations on the detector of calibration features may have changed by several tens of pixels, rotation and distortion may be present as well as simple translation. We will refer to this scenario as "format recovery". A sparse line list with easily identifiable features is required in order to avoid false matches, but saturation of lines and blending that can lead to inaccuracies at the 1 pixel level are not so critical in this scenario. At the other extreme, the final stage of optimising the wavelength solution occurs repeatedly during routine operations of a stable instrument. Here

Further author information: (Send correspondence to Paul Bristow)

Paul Bristow: E-mail: bristowp@bristowp@eso.org, Telephone: +49 8932006454 
one can expect that the spectral format is only changing at the sub-pixel level, the previously valid wavelength solution can reliably be used to locate lines, but a highly accurate fit is sensitive to errors resulting from blends and saturated lines.

Murphy et $\mathrm{al}^{2}$ describe a sophisticated approach to the latter problem that uses a bootstrapped comparison between wavelength calibration data from an operational instrument (UVES on the VLT) and a comprehensive catalogue in order to derive a list of lines that are useful for UVES calibration. We take a similar approach here using early laboratory data from the medium resolution spectrograph X-shooter ${ }^{11}$ (specifically the subspectrograph for the visible wavelength range from $550 \mathrm{~nm}$ to $1 \mu \mathrm{m}$ known as VIS), but concentrate on the former problem, the format recovery scenario. In this case we need a list of isolated features that cannot easily by falsely matched to a near neighbour.

A crucial factor that determines our requirements and leads us to a different approach from previous solutions to this problem is our model for the wavelength solution. Rather than applying the traditional approach of applying independent polynomials to each of the Echelle spectral orders, we adopt a physical model of the instrument. The physical model approach is described in more detail in Bristow et al $2008 \mathrm{a}^{3}$ (see also Bristow et al $2008 \mathrm{~b}^{4}$ for the $\mathrm{X}$-shooter specific implementation). The parameters of this model are optimised to fit reference lines from the entire spectral range simultaneously. As a result we do not require a certain minimum number of reference line identifications per order as classical polynomial wavelength solutions do (typically 5-10), so long as they sample the detector space uniformly. Moreover optimising the parameters for the physical model requires a relatively low total number of reference line identifications. However, model parameter optimisation is very sensitive to falsely identified reference lines.

Bearing all of the above in mind, we set out to devise such a method that would fulfill the following criteria:

1. The signal to noise probable in realistic calibration exposures should be taken into account.

2. Bright lines should not be rejected on the basis of very faint near neighbours.

3. It should be possible to adjust the window size in which the line is guaranteed to be unique.

A simplistic approach to avoiding false matches is to select only those lines in our master catalogue that are isolated in the sense that there are no significant neighbours within a given radius. This conservative approach can result in relatively few lines in our customised lists, but our physical model does not require so many calibration features in order to optimise its parameters as a classical polynomial solution would to fit its coefficients. In what follows we define what constitutes a significant neighbour and describe a method for performing this kind of selection for any "isolation" radius.

\section{METHOD}

\subsection{Choice of master catalogue and pre-selection}

The choice of master catalogue is not critical here. For Th-Ar we follow Murphy et al ${ }^{2}$ in constructing a catalogue from the Th catalogue of Palmer and Engleman ${ }^{5}$ and the Ar catalogues of Whaling ${ }^{6,}{ }^{7}$ For pen ray lamps we obtained input catalogues from the NIST Atomic Spectra Database. ${ }^{9}$ We rejected lines from contaminant species, known blends and uncertain identifications.

\subsection{Selection of lines for custom lists}

In order to perform the selection we require a wavelength solution that permits us to make an accurate wavelength calibrated 1D extraction from calibration source exposure. This is clearly a "chicken and egg" paradox. However, our aim is to generate a line list that will enable the automatic matching of lines in the format recovery scenario. It is quite possible that the very first wavelength calibration has been arrived at via interactive identification of lines, indeed this is the current situation for X-shooter. Moreover, the process described below could be carried out using simulated calibration source exposures (via the physical model) as discussed in section 2.4.

We proceed as follows: 
1. The physical model based wavelength solution is used to extract and wavelength calibrate a $1 \mathrm{D}$ spectrum for each order. This is median filtered to remove background and continuum.

2. A list of centroid locations of features in the 1D spectra are identified via convolution with a Gaussian of appropriate FWHM.

3. These centroid locations are identified with reference wavelengths in the master catalogue.

4. For each centroid in the list we examine the neighbouring pixel bins in the $1 \mathrm{D}$ extracted spectrum in order to determine how "isolated" the feature is. We use a novel and complex definition of isolation to be isolated within $N$ pixels:

$$
\begin{gathered}
\left\|C o G\left(F,-W_{\text {min }}: W\right)\right\|<P \forall W_{\text {min }}<W<=N \\
\left\|C o G\left(F,-W: W_{\text {min }}\right)\right\|<P \forall-N<=W<-W_{\text {min }}
\end{gathered}
$$

where the function $C o G$ is the centre of gravity of a section of the extracted 1D spectrum $F$ between bins $X_{1}$ and $X_{2}$ :

$$
\operatorname{CoG}\left(F, X_{1}: X_{2}\right)=\frac{\sum_{X=X_{1}}^{X=X_{2}} F[X]\left(X-C_{\text {list }}\right)}{\sum_{X=X_{1}}^{X=X_{2}} F[X]}
$$

$C_{\text {list }}$ is the list centroid from step $2, W_{\min }$ is the core radius in pixels expected for individual spectral lines on the spectrograph detector and $P$ is the tolerance in pixels. After some experimentation we found the best results for the X-shooter VIS arm with $P=\max (0.2, N / 40)$ This definition is illustrated in figure 1. It can be interpreted as requiring that flux from within $N$ pixels (the "isolation width") does not distort the centre of gravity of the feature itself. If the feature itself has a low $\mathrm{S} / \mathrm{N}$ then noise in neighbouring bins alone may be enough to distort the centroid. At the other extreme, a very strong feature may not have its centroid distorted by nearby faint features. Generally however, the centroid of a feature will be distorted beyond the tolerance if another real feature is present inside the window.

5. Features fulfilling these criteria have their wavelengths and measured flux inserted in the custom list. Each list is then specific to (and only applicable to) the value of $N$ used, to the lamp used, to the instrument (here always $\mathrm{X}$-shooter VIS) and to the $\mathrm{S} / \mathrm{N}$ of the arc lamp exposure.

\subsection{Application of custom lists}

The lines in the custom lists selected as described above are isolated to within $N$ pixels. This means that in the format recovery scenario where the maximum distortion suffered by any location on the detector is $\leq N / 2$ pixels, we can safely search for the centroid in a window of radius $N / 2$ pixels around the previously known centroid for the line and we can be sure to match the line uniquely. We verified this claim by applying transformations to the Th-Ar extracted 1D spectra for each order that resulted in up to $N / 2$ pixel shifts in the centroids for each of the values of $N$ in table 1 . We then used the Th-Ar lists for the corresponding value of $N$ to determine which lines should be matchable. The wavelengths in the list were converted to expected positions based upon the original wavelength solution (via the physical model) that was valid before the transformation. Then we computed the centroid in a window of width $N$ centered on the expected position. The centroid calculated this way was indeed always within $P$ pixels of the transformed feature position corresponding to the correct wavelength. 


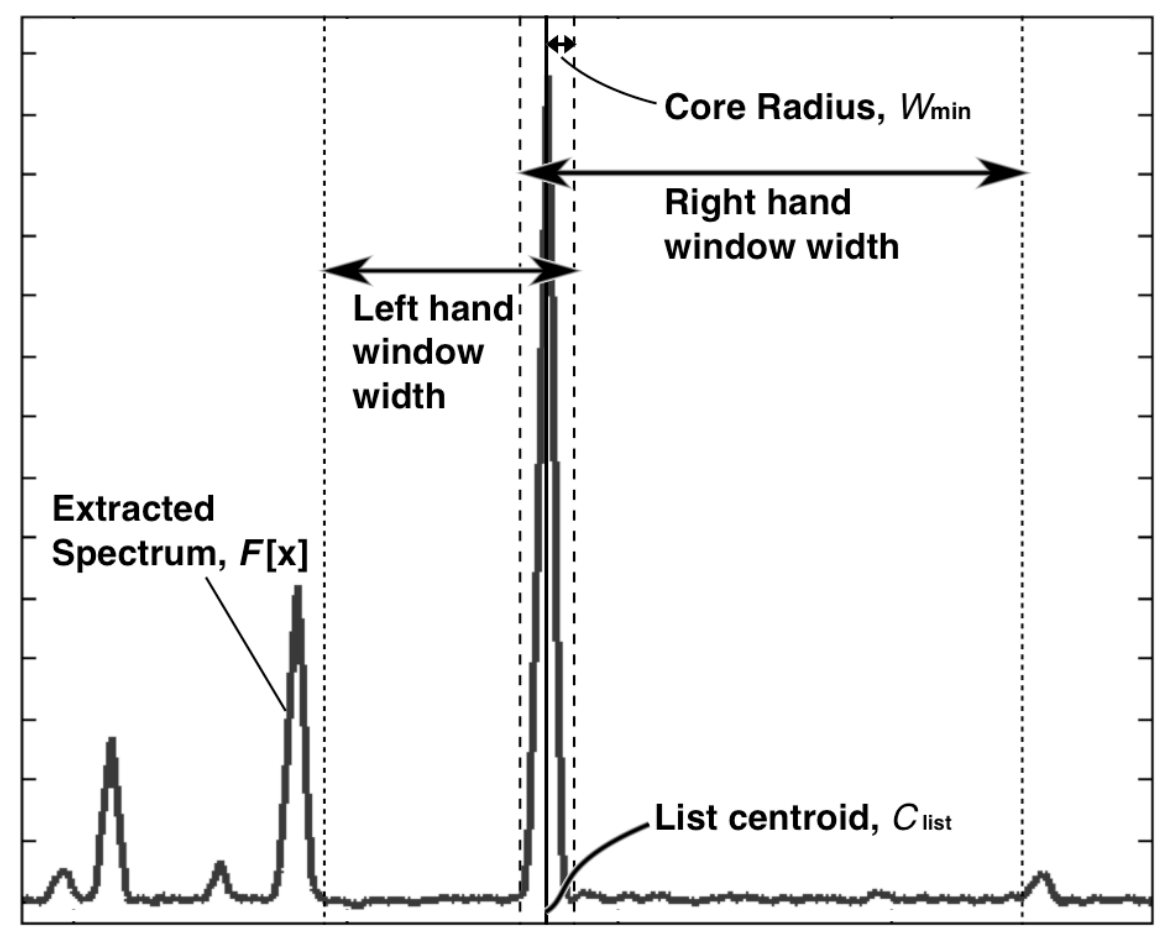

Figure 1. Schematic representation of our criteria for isolation. First a window encompassing the feature (entirely) and an extended area to the right is constructed. Next the centre of gravity for this window is computed and compared to the list centroid from step 2 (see main text). If the offset is within a given tolerance then the window is extended further to the right and the comparison is repeated until the offset exceeds the tolerance. The window size is only shown in the figure at its maximum extent before the test fails. The procedure is the repeated on the left. If the smaller of the two window sizes (on the left in this example) exceeds the minimum isolation value for the list, $N$, then it is selected.

\subsection{Use of simulated data}

It is in principle possible to use the physical model to create realistic synthetic X-shooter arc lamp exposures and apply the method described above to the synthetic data. In this way we can create the lists before the instrument is even built, solving the "chicken and egg" problem mentioned earlier. However, in practise we have found that, even the best available wavelength reference standard catalogues are not adequate for this approach. Subtle differences can lead to crucial mistakes in the inclusion and omissions of lines compared to what one finds with the real data. Moreover, if the input catalogue for the 2D simulated data (from which the $1 \mathrm{D}$ spectrum is extracted) is not sufficiently deep, lines will appear isolated when they are not. By using real exposures we avoid this pitfall, we then use the input catalogue only to find the wavelength of selected features. For the remainder this discussion then, we concentrate on lists generated from real data. Meanwhile Kerber et $\mathrm{al}^{1}$ illustrate what can be achieved through modelling in anticipation of the first real data. Moreover, the pipeline wavelength calibration for the CRIRES instrument, which also makes use of a physical model, uses automatically generated synthetic data to derive custom line lists for the fine tuning of the physical model.

\section{RESULTS}

\subsection{Th-Ar hollow cathode lamp}

We used the method described to select Th-Ar custom line lists for a range of values of $N$ for early Th-Ar HCL exposures obtained in December 2007 and March 2008. Table 1 shows the number of lines selected for each value of $N$. Figure 2a shows a section of order 23 from the March 2008 data in which lines selected for several values of $N$ are highlighted. (The different values for the two data sets will become apparent). 

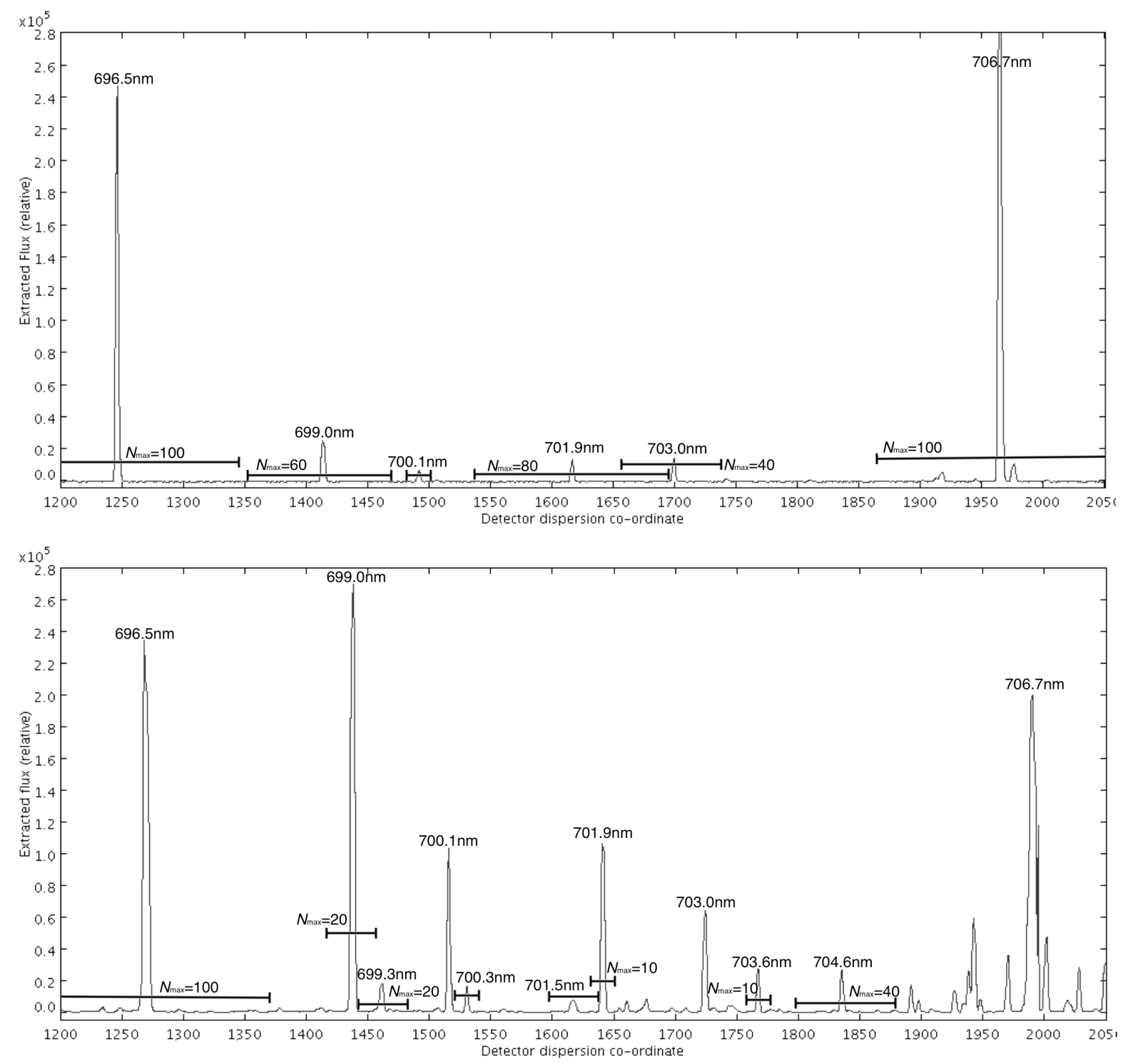

Figure 2. Extracted flux plotted against detector dispersion co-ordinate for order 23 of a VIS exposure of a Th-Ar HCL source, above is data from December 2007, below from March 2008. Note that the relative strengths of the lines have changed. Also indicated are the maximum values of the isolation parameter $N$ for which some of the calibration features fulfilled our selection criteria, the range within which the lines are considered isolated is shown by the error bars. 
Table 1. The Number of lines selected for various values of $N$ and the available X-shooter arc lamps.

\begin{tabular}{cccccccc} 
& \multicolumn{2}{c}{ Th-Ar HCL } & \multicolumn{4}{c}{ Pen ray lamps } \\
$N$ & (Dec'07) & (Mar'08) & Ar & Kr & Ne & Xe \\
\hline 10 & 207 & 233 & 51 & 47 & 92 & 27 \\
20 & 117 & 83 & 32 & 34 & 77 & 18 \\
40 & 48 & 18 & 27 & 25 & 68 & 14 \\
60 & 35 & 7 & 25 & 16 & 56 & 11 \\
80 & 16 & 3 & 22 & 15 & 45 & 11 \\
100 & 11 & 2 & 18 & 14 & 43 & 9 \\
\hline
\end{tabular}

The total number of isolated lines for a given value of $N$ is important, but so too is their distribution, we require good coverage of the detector array. Figures 3a (December 2007 data) and b (March 2008 data) indicate the positions of the selected lines on the detectors. Where a line is present in just the $N=10$ list it has just one circle, where it is included in the $N=10$ and $N=20$ lists it has 2 circles and so on up to 6 circles if it occurs in all the lists up to $N=100$.

Between the epochs of the two data sets there had been some significant changes in the instrument configuration causing a change of spectral format, transforming the location of some lines by up to 40 pixel, i.e at the time that the March 2008 exposures were taken we were in the format recovery scenario, using the physical model with parameters optimised with the December 2007 data we could only be expected predict the location of reference wavelengths to within 40 pixels of where they would actually be.

From table 1 it is clear that for $N=80$ (allowing a maximum shift of $N / 2=40$ ) we do not have many lines, probably too few even for the relatively lenient requirements for optimising the physical model parameters. Indeed this is a problem for the Th-Ar HCL spectrum when used for automatic identification of lines after a large change in spectral format, the spectrum is too rich and isolated lines are hard to find.

Nevertheless, as a test of the method we intended to use the December list to automatically identify the 16, $N=80$, December 2007 lines in the March 2008 data. However we found that there were in fact some false matches. It turns out that the two data sets were obtained with the Th-Ar lamp operating at different currents, for the March 2008 data a higher current was used (unfortunately we do not have a record of the exact current levels used since the data was taken during an early stage in testing at which time such details were not automatically logged in the FITS headers). The lower panel in Figure 2 shows the same wavelength range as the upper panel but for the March 2008 data. It is clear that the relative strengths of the lines have changed resulting in different lines being identified as isolated at the various values of $N$. The change in relative strengths of Th-Ar HCL line species is a well known phenomenon (see for example Kerber et al $2008^{10}$ ). In order to use this method for Th-Ar HCL exposures then, it would be necessary to operate the lamp at a constant current setting. We do not yet have data at the same current setting for two epochs spanning a major change in the instrument spectral format.

\subsection{Pen Ray lamps}

X-shooter has 4 "pen ray" lamps (Neon, Argon, Krypton and Xenon), originally intended for calibration of the near infra red arm. We applied our custom line list selection to exposures made with these lamps (only available for the March 2008 epoch). The results are displayed in table 1 where we see that, though for low values of $N$ these lamps have many less isolated lines than the Th-Ar HCL, as $N$ increases the number of isolated lines drops off less rapidly. This is an obvious consequence of the sparser spectrum of these lamps compared to the Th-Ar HCL. In particular the Ne spectrum has many isolated lines right out to $N=100$ (distortions of up to 50 pixels). Figure 4 is a graphical representation of table 1 making clear the strong dependence upon $N$ for Th-Ar and the weaker dependence for the pen rays. 

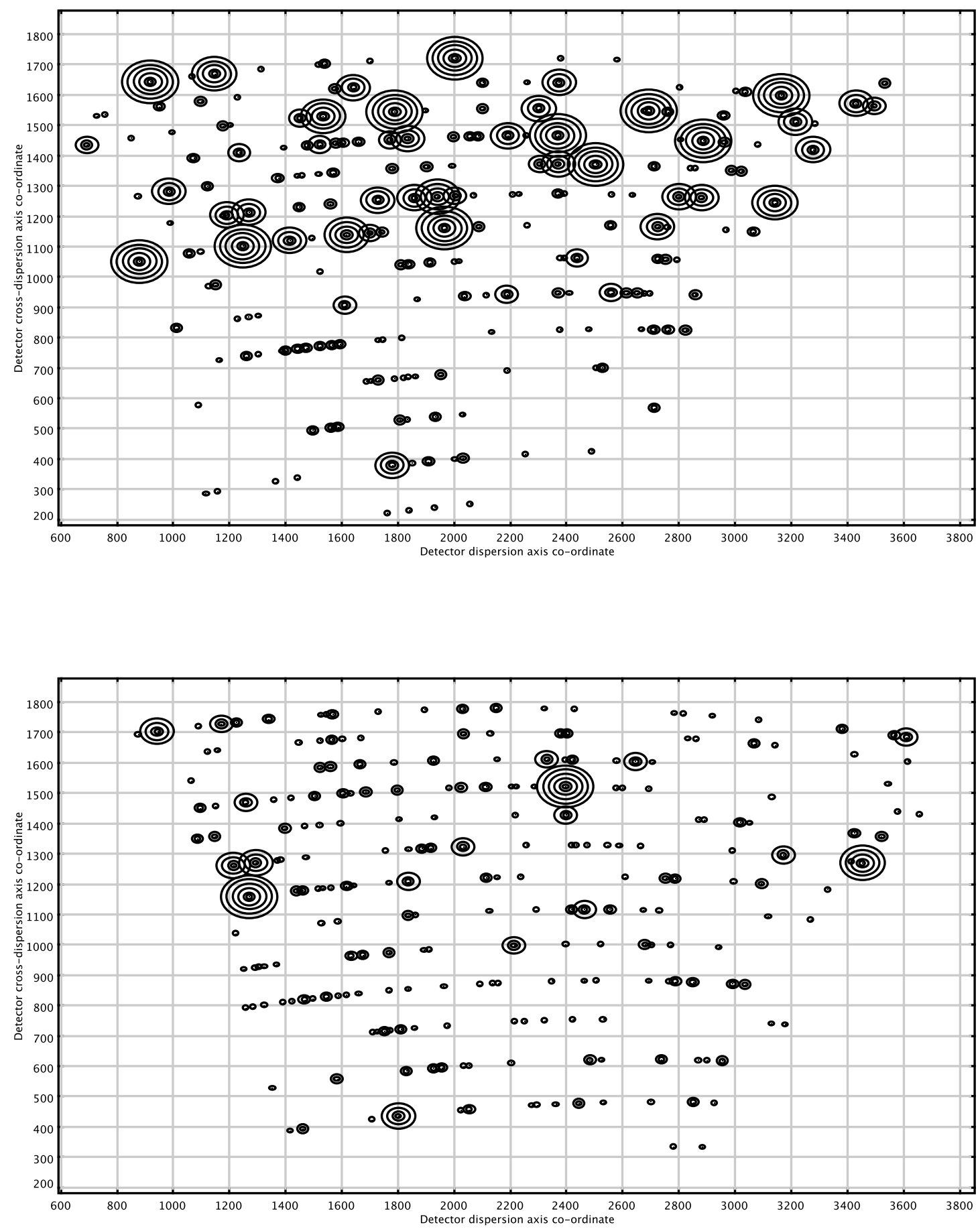

Figure 3. Location on the VIS detector array for lines selected in lists with the isolation parameter, $N$ taking the values in table 1 for the Th-Ar HCL (above: December 2007 data; Below: March:2008 data). The size of the circles reflects the value of $N$, thus a line that is present in, for example, the lists up to $N=60$ will have 4 co-centric circles for $N=10,20,40$ and 60 . 


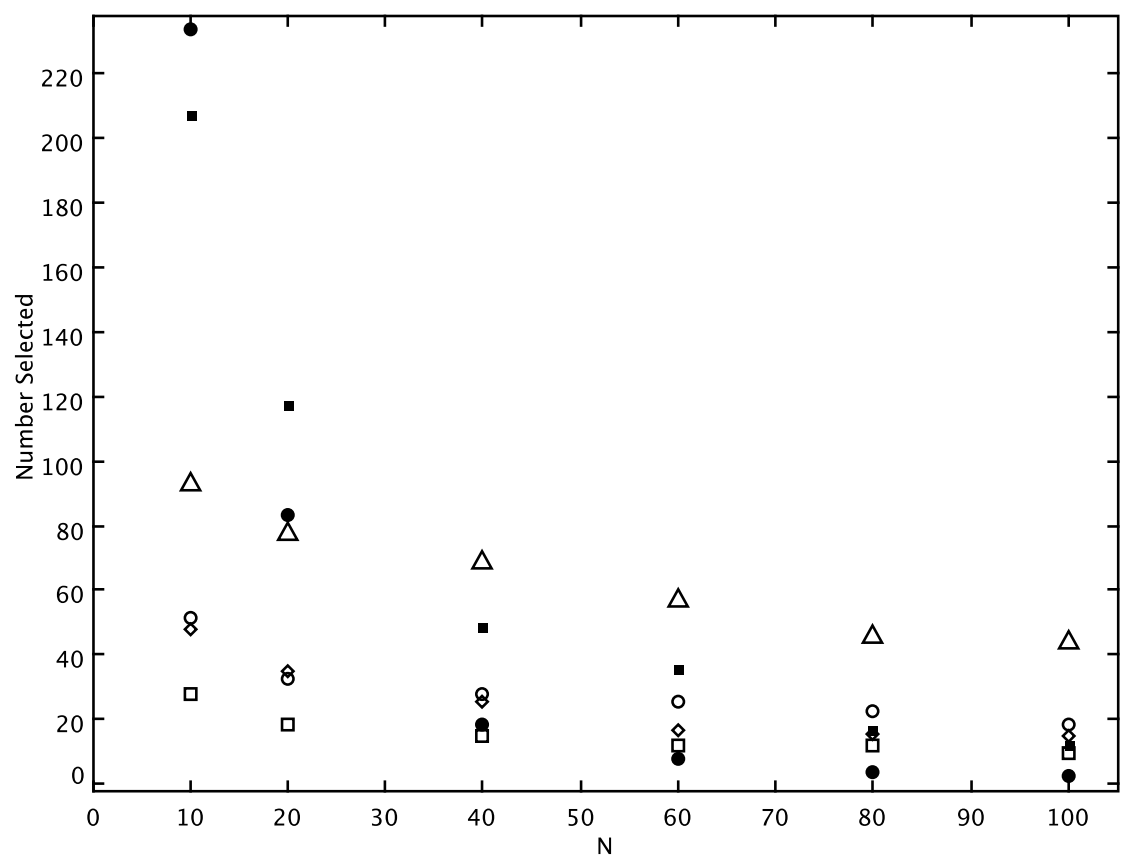

Figure 4. The Number of lines selected for the available X-shooter arc lamps as a function of isolation witdh $N$. Key: solid circles=Th-Ar HCL (Mar'08); solid squares=Th-Ar HCL (Dec'07); open circles=Ar; open diamonds=Kr; open triangles $=\mathrm{Ne}$; open squares $=\mathrm{Xe}$

Figures 5 and 6 show the distribution of selected features on the detector for Ar (top) and $\mathrm{Kr}$ (bottom) and $\mathrm{Ne}$ (top) and Xe (bottom) respectively.

\section{DISCUSSION}

On the basis of the above analysis, Ne would seem to be the best single source when using this method for selecting wavelength standards for the purpose of recovering the wavelength solution after a major ( $\sim 40$ pixels) change in spectral format. The $>40$ lines selected even for $N=100$ have good coverage on the detector and are enough to constrain the physical model parameters so that the physical model will predict the location of lines to within 3 pixels across the detector. At this stage much deeper line lists can be used to refine the model further.

We could increase the effective number of lines at high $N$ by combining the results from separate pen ray lamp exposures (most likely just $\mathrm{Ar}$ and $\mathrm{Ne}$, since $\mathrm{Kr}$ and Xe simply don't have many features at any value of $N)$. It is important to remember however that a single exposure of several pen ray lamps simultaneously would not be a good idea since this would change the flux distribution and lines that were isolated in the individual exposures may well no longer be isolated.

Regarding the Th-Ar HCL, clearly the current setting used for the December 2007 data gives a spectrum that has more lines passing our selection criteria at higher values of $N$. However, if we assume that Ne is the best source for the format recovery scenario, then operating the Th-Ar HCL at the lower current setting will provide a richer spectrum of fainter Th lines useful for the fine tuning of the model parameters during routine operations.

Signal to noise also plays a role in our results. It is clear from figure 2 that the data from the two epochs have different S/N. The December 2007 data was a 30s exposure, while the March 2008 data was just 4s. This 

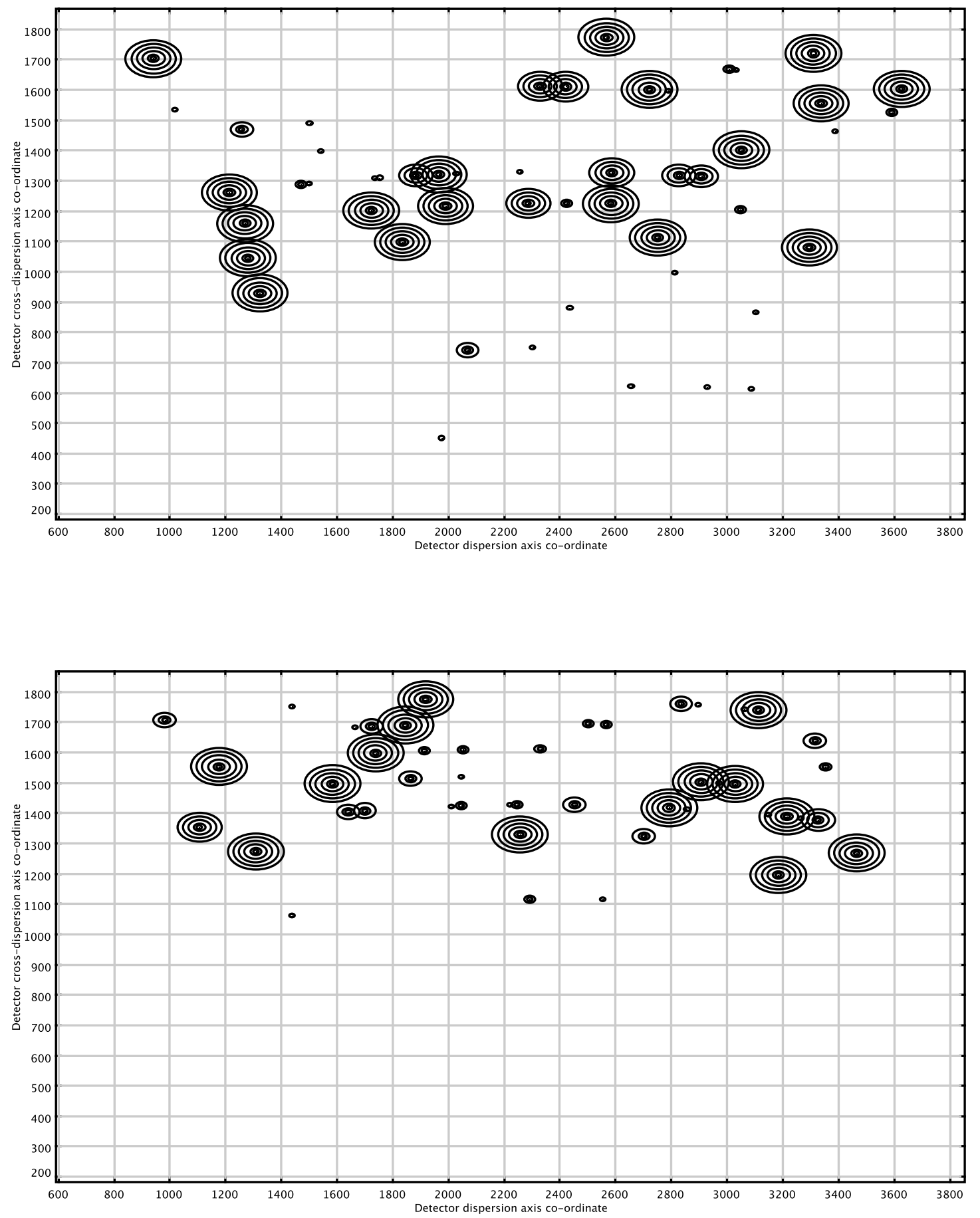

Figure 5. As figure 3 but for the Ar (above) and Kr (below) pen ray lamps. 

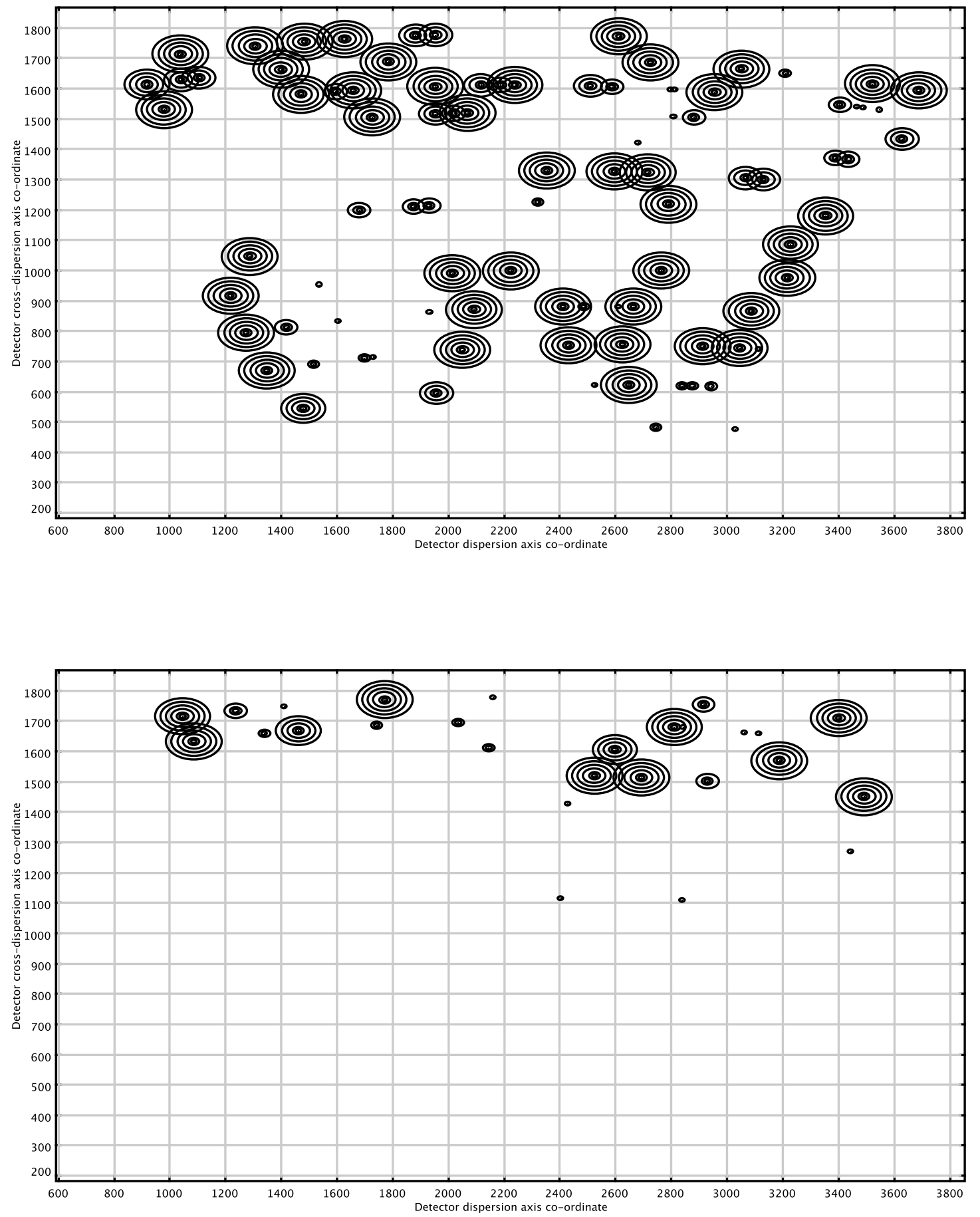

Figure 6. As figure 3 but for the Ne (above) and Xe (below) pen ray lamps. 
obviously does not explain the difference in relative line strengths, that can only be explained by the current setting. However an increased S/N in the March 2008 data set would have possibly lead to more faint features having sufficiently robust centroids to pass our selection criteria. This is unlikely though to increase the number of lines selected for high $N$ since crowding would be increased rather than reduced. In general the selection procedure is designed so that it is not sensitive to the $\mathrm{S} / \mathrm{N}$.

We have restricted our analysis here to X-shooter VIS data. The situation is likely to be qualitatively similar for the UVB arm. A preliminary analysis reveals that the Th-Ar HCL spectrum is even more crowded in the UVB range, strengthening the need for pen ray exposures to aid an automatic approach to the recover format scenario. The NIR arm on the other hand has a less crowded Th-Ar HCL spectrum, see Kerber et al $2008 .{ }^{1}$

\section{SUMMARY}

We have considered the problem of down selecting catalogues of wavelength standards for commonly used arc lamps in order to obtain lists of lines whose centroid will be reliably found within a window of a given width. The method that we have developed is conservative returning a relatively short list of lines with robust centroids. It is intended for use in the scenario where the locations on the detector of calibration features may have changed by an amount much larger than the characteristic size of calibration lines as they appear on the detector due to a physical intervention to the instrument or earthquake damage. Since we use a physical model to drive our wavelength solution we do not have strict requirements for the total number of lines or the minimum number of lines in each order (compared to a classical polynomial wavelength solution). However we do have the requirement that there are no false matches and that the detector space is well sampled. Whilst our method is intended to be a widely applicable solution, our analysis has been for the visual wavelengths arm of the X-shooter detector.

The method that we have developed works best with pen ray lamps which produce a less crowded spectrum than the alternative Th-Ar HCL. From a single Ne exposure our method will produce $>40$ lines whose centroids are robust within 100 pixels, allowing the recovery in the case where the locations on the detector of calibration features may have changed by up to 50 pixels. This customised list of lines allows optimisation of the model parameters so that the model is able to predict line positions to within 3 pixels. At this stage the format has essentially been recovered and different criteria are needed to select lines for lists to be used in further refining the model parameters.

\section{REFERENCES}

[1] F. Kerber, F. Saitta, P. Bristow, J. Vernet, "Wavelength calibration sources for the near infrared arm of $\mathrm{X}$-shooter", These proceedings

[2] Murphy, M. T.; Tzanavaris, P.; Webb, J. K.; Lovis, C., MNRAS, 378, 1, p 221-230 (2007)

[3] P. Bristow, F. Kerber, M. R. Rosa, "Advanced Calibration Techniques for Astronomical Spectrographs", The Messenger, 131, p2 (2008)

[4] P. Bristow, F. Kerber, M. R. Rosa, "The X-shooter Physical Model", These proceedings

[5] Palmer B.A. \& Engleman R., "Atlas of the Thorium Spectrum", Los Alamos National Laboratory, Los Alamos (1983)

[6] Whaling et al., J. Quant. Spectrosc. Radiat. Transfer, 53, p1 (1995)

[7] Whaling et al., J. Res. Natl. Inst. Stand. Technol., 107, p149 (2002)

[8] P. Bristow, F. Kerber, M. R. Rosa, "Advanced 2D Spectroscopic Predicted Data", These proceedings

[9] Ralchenko, Yu., Kramida, A.E., Reader, J., and NIST ASD Team. NIST Atomic Spectra Database (version 3.1.5), [Online]. Available: http://physics.nist.gov/asd3 [2008, May 26]. National Institute of Standards and Technology, Gaithersburg, MD. (2008)

[10] Kerber, F., Nave, G., Sansonetti, C.J., Bristow, P., Rosa, M.R.: "The Spectrum of Th-Ar Hollow Cathode Lamps in the 900-4500 nm Region: Establishing Wavelength Standards for the Calibration of VLT Spectrographs" in The Future of Photometric, Spectrophotometric Polarimetric Standardization, ed. C. Sterken, ASP Conf. Ser. 364, p. 461 (2007)

[11] Vernet, J. et al "Coming Soon on Stage: X-shooter", The Messenger, 130, 5 (2007) 